\title{
Is adult cardiac regeneration absent in Xenopus laevis yet present in Xenopus tropicalis?
}

\author{
Lindsey Marshall, Fabrice Girardot, Barbara A. Demeneix and Laurent Coen* (i]
}

\begin{abstract}
We recently used an endoscopy-based resection method to explore the consequences of cardiac injury in adult Xenopus laevis, obtaining the result that the adult Xenopus heart is unable to regenerate. At 11 months postamputation, cellular and biological marks of scarring persisted. We thus concluded that, contrary to urodeles and teleosts, adult anurans share a cardiac injury outcome similar to adult mammals. However, in their work published in this journal on the 13 December 2017, Liao et al. showed that the adult Xenopus tropicalis heart is capable of efficient, almost scar free regeneration, a result at odds with our previous observation. These findings contrast with and challenge the outcome of adult heart repair following injury in Xenopus species. Here we discuss the question of the intrinsic cardiac regenerative properties of an adult heart in anuran amphibians.
\end{abstract}

Keywords: Anuran amphibians, Xenopus laevis, Xenopus tropicalis, Cardiac regeneration, Heart injury

Open discussion on the reproducibility of adult Xenopus heart regeneration data following apical resection:

Dear Editor,

A key question in cardiovascular biology is to what degree the heart is able to regenerate after tissue damage, and why this capacity varies between evolutionaryseparate vertebrate species. If teleost fishes (with the exception of medaka) display high regenerative capacities throughout their entire life, why is mammalian heart regeneration hindered soon after birth, causing adults to lose the ability to regenerate their injured myocardium. In amphibians, evolutionarily positioned between these two phyla, urodeles (i.e. newt, axolotl or salamander) possess lifelong cardiac regenerative capacity like zebrafish, whereas anuran amphibians (i.e. Xenopus) remained poorly investigated till recently [1].

In the past year, we and others published contradictory results on the cardiac outcome in adult anuran

\footnotetext{
*Correspondence: coen@mnhn.fr

Evolution des Régulations Endocriniennes, Département Adaptation du vivant, UMR CNRS 7221, Muséum National d'Histoire Naturelle, Sorbonne Université, Paris, France
}

amphibians after cardiac resection [2, 3]. Following an apical amputation of $4 \%$ of the ventricle volume in adult Xenopus laevis, we observed significant fibrous scarring, cardiomyocyte hypertrophy and sarcomere disorganisation near the injury site, persisting 11 months postamputation. Together, these observations allowed us to conclude that the adult heart was unable to regenerate in this species [2]. Conversely, in the closely related species Xenopus tropicalis, a cardiac apex resection removing approximately $10 \%$ of the adult heart length, showed almost scar-free regeneration in 30-60 days, which provides, according to the authors, "a powerful tool for recapitulating a perfect regeneration phenomenon" [3]. These contrasting results could be interpreted as indicating that cardiac regenerative capacity is differentially distributed in the Xenopus genus.

It is not the first time that heart regeneration observations noted in the literature are controversial. Such an example was reported when analysing cardiac regeneration in the MRL/MpJ mouse strain, where some groups observed cardiac regeneration in adult MRL mice whilst others did not, but this discrepancy remains for the moment unresolved (see in [1]).

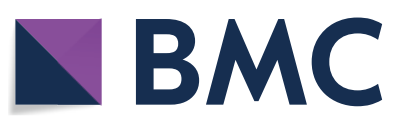

(c) The Author(s) 2018. This article is distributed under the terms of the Creative Commons Attribution 4.0 International License (http://creativecommons.org/licenses/by/4.0/), which permits unrestricted use, distribution, and reproduction in any medium, provided you give appropriate credit to the original author(s) and the source, provide a link to the Creative Commons license, and indicate if changes were made. The Creative Commons Public Domain Dedication waiver (http://creativecommons.org/ publicdomain/zero/1.0/) applies to the data made available in this article, unless otherwise stated. 
However, the most pertinent example that has been the cause of confusion and conflicting positions comes from observations in neonate mice, a topic that was "hotly" debated after experimental results showed a different outcome in cardiac rebuilding [4]. In 2011, Porrello et al. [5] published the elegant demonstration that neonatal mice have the potential to fully regenerate resected myocardium in 21 days, whereas a study by Andersen et al. [6] called into question these results. This paper reported limited evidence of regeneration in apically resected neonate hearts at 21 days post-surgery [6]. The conflicting results were partly resolved by Bryant et al. [7], who advanced technical considerations as an important variable that could influence the experimental design, and may explain failure to reproduce the observations between researchers applying a similar cardiac injury protocol. However, the question still remains unanswered, notably in the light of recent work by Zebrowski et al. [8] that fuels the debate. These authors showed that binucleation and cell-cycle arrest occur very soon after birth and are boosted following heart resection in newborn mice [8]. They suggest that if there is a regenerative period in the mammalian neonate, it is very short.

It is well known that mammalian cardiomyocytes become binucleated and polyploid soon after birth, resulting in cell-cycle arrest and hypertrophy in adults [8-10]. In contrast, in zebrafish, cardiomyocytes remain mostly mononucleated even at adult stage, potentially explaining why they continue to be highly proliferative throughout life [10]. While cardiomyocyte nucleation has been poorly investigated during Xenopus development, a major difference between $X$. laevis and X. tropicalis is that the first has a pseudotetraploid genome and the latter a diploid genome. This difference might be implicated in the opposite outcomes of their cardiac regenerative capacity observed in adults. Potentially, the tetraploid nature of cardiomyocytes in $X$. laevis may render them more prone to polyploidy and hypertrophy. In contrast, $X$. tropicalis could retain a higher concentration of mononucleated cardiomyocytes, similar to zebrafish, which may explain the proliferative response observed in adult heart.

Bryant and co-authors have shown that the resection size influences the extent of scarring and thus the regenerative outcome of the mouse heart [7]. However, we believe that resection size cannot explain the discrepancies between results in $X$. tropicalis observed by Liao and collaborators and our own observations in adult $X$. laevis. They report that their protocol results in the removal of approximately $10 \%$ of ventricle length while our approach led to the removal of $4 \%$ of the ventricle volume, hence, one would rather expect our protocol to be more favourable to regeneration.
A major difference in our respective approach is the technical methodology applied to perform the resection. We developed a minimally invasive endoscopy-based resection method to explore the consequences of cardiac injury in adult frogs, while in Liao's work, the cardiac apex was resected using Vannas scissors after externalising the heart. Again, it could be expected that our approach leads to less trauma to cardiac tissue and should also favour regeneration. Nonetheless, we have also performed resection applying a protocol similar to Liao's to X. laevis frogs and observed no evidence of cardiac regeneration (unpublished experiments). Another difference between both techniques was that, following tissue removal using biopsy forceps via an endoscope, we do not apply pressure to the wound in the form of sterile cotton, due to the internal progress of the endoscopic technique. Thus, in our technique, the blood flow immediately following amputation remained internally trapped within the animal. Yet again, we do not think that this modification underlies the different results, as we observed a rapid formation of a large blood clot at the injury site after the biopsy amputation, similar to Liao's description which mentioned the formation of the clot in few seconds. Furthermore, the clot is limited to the site of amputation as the pericardium is only slightly open at the apex and therefore remains close around the heart during and after the amputation, protecting the heart from the external environment (which is not the case in Liao's method). Despite these reservations, it is still possible that the methodology, endoscopy versus external heart resection could be implicated in the differential responses.

We consider the critical point could be the methods used to determine the presence (or absence) of cardiac regenerative capacity, notably, how the conclusion of regeneration is obtained (monitoring fibrosis extent, cardiomyocyte hypertrophy, cardiomyocyte cell-cycle activity...) as underlined by Bryant et al. [7], in their discussion on the mouse model. Thus, in our respective work on Xenopus, the distinct criteria used to assess the regenerative process, may explain at least in part the observed differences. In our paper, we not only performed histological analyses, but we also made extensive use of immuno-labelling to assess the degree of fibrosis, hypertrophy and the sarcomere structure. All criteria considered converged to the same conclusion that regeneration is totally absent in Xenopus hearts in adult frogs even after about 1 year post amputation [2]. While Liao's work mainly relies on histological staining, it is noteworthy that the fibrosis is indeed absent in most of their samples 60 days post injury [3].

Another disputable point is the methods used by Liao and collaborators to assess cardiomyocyte mitoses, that 
according to some authors is suggestive of a regenerative capacity [5, 11]. Liao et al. first used hematoxylin/ eosin staining. In our opinion, and given the available stringent and more adapted methodology to detect newly formed cardiomyocytes (for instance, using pulse/chase experiments with BrdU incorporation to detect DNA synthesis in cardiomyocyte nuclei $[5,11])$, we consider that hematoxylin/eosin staining is not sufficiently precise to follow proliferation during regeneration and risks over estimating the proliferative status of cardiomyocytes. When analysing cardiac regeneration in zebrafish, hematoxylin/eosin staining has been used to observe the tissue as a whole and to show the presence of nucleated blood cells in the damaged area following resection [12]. Thus it cannot be excluded that the proliferative cells identified by Liao et al. using hematoxylin/eosin staining could have represented proliferating non-cardiomyocyte cells, such as erythrocytes or immune cells rather than cardiomyocytes.

Liao et al. also used another method to detect cardiomyocyte mitoses. They performed co-labelling using the cytoplasmic alpha-skeletal muscle actin $(\alpha-S M A)$ antibody to label cardiomyocytes and the $\mathrm{pH} 3$ antibody to follow cell cycle activity (proliferation). We are surprised that authors did not use the more appropriate marker Mef2c (Myocyte Enhancer Factor $2 \mathrm{C}$ ), that specifically labels the cardiomyocyte nuclei, to identify unambiguously the co-localised pH3 positive cardiomyocytes in their samples. Thus, the antibody combination used by Liao et al. to detect proliferative cardiomyocytes can lead to misinterpretation, especially because a number of other non-cardiomyocyte cells, such as blood cells, fibroblasts and epicardial cells also proliferate after cardiac injury.

Transgenic approaches can be also used to specifically label cardiomyocyte nuclei, for instance, using a nuclear localised fluorescent reporter under control of a cardiac specific promoter. Such protocols have been applied to zebrafish, using the cardiac myosin light chain 2 (cmlc2) promoter to differentiate actual cardiomyocytes from non-cardiomyocyte cells, at the amputated site after cardiac resection [13]. A similar approach could be used in Xenopus, for instance, by creating transgenic animals with a nuclear fluorescent reporter driven by the cardiac specific promoter pMLC1v [14]. Using such a transgenic approach would allow easy and accurate labelling of cardiomyocyte nuclei. It would thus facilitate the unambiguous identification of the cells that proliferate after cardiac amputation in an adult frog. Furthermore, transgenic animals have also been used to assess the origin of proliferating cardiomyocytes in zebrafish and neonatal mice $[5,11]$. Here, an inducible Cre recombinase system driven by a cardiac specific promoter was used to de-repress a reporter construct (GFP in zebrafish; lacZ in mice) blocked by a loxPflanked transcriptional stop codon, allowing expression of the reporter specifically in resident cardiomyocytes. After amputation, the reporter expression was observed in newly formed cardiomyocytes that replaced the lost myocardial tissue. These demonstrations showed that for neonatal mice and zebrafish, myocardial regeneration is principally achieved by newly formed cardiomyocytes derived from preexisting cardiomyocytes $[5,11]$. Applying a similar technology in Xenopus seems possible as it has been shown that both inducible system and Cre-based approaches can be used in this species $[15,16]$. Developing such methodologies would allow lineage tracing in the heart and allow one to determine the origin of newly formed cardiomyocytes after cardiac amputation in Xenopus.

Finally, differences in cardiac regenerative capacity have previously been observed in organisms belonging to the same group. Indeed, in teleosts, zebrafish possess lifelong and highly efficient cardiac regenerative capacity [12] while this competence is not observed in medaka [17]. Therefore, it is not necessarily surprising that closely related Xenopus laevis and tropicalis may have different and opposed cardiac outcomes following heart resection. In teleosts, distinct activation of the immune response is involved in these differences in heart repair [18]. Remarkably, proper activation of the immune system is also required for cardiac regeneration in the adult urodele amphibian axoltl [19] as well as for determining competence for tail-regeneration and scar-free wound healing in X. laevis [20,21]. It would hence be extremely interesting to explore whether adult Xenopus laevis and tropicalis species also display differences in their immune responses following cardiac injury.

Authors' contributions

All author contributed equally to this letter. All authors read and approved the final manuscript.

\section{Acknowledgements \\ Not applicable. \\ Competing interests \\ The authors declare that they have no competing interests.}

Availability of data and materials

Not applicable.

Consent for publication

Not applicable.

Ethics approval and consent to participate

Not applicable.

Funding

Not applicable. 


\section{Publisher's Note}

Springer Nature remains neutral with regard to jurisdictional claims in published maps and institutional affiliations.

Received: 4 January 2018 Accepted: 16 April 2018

Published online: 19 April 2018

\section{References}

1. Vivien CJ, Hudson JE, Porrello ER. Evolution, comparative biology and ontogeny of vertebrate heart regeneration. NPJ Regen Med. 2016;1:16012

2. Marshall L, Vivien C, Girardot F, Pericard L, Demeneix BA, Coen L, Chai N. Persistent fibrosis, hypertrophy and sarcomere disorganisation after endoscopy-guided heart resection in adult Xenopus. PLOS ONE. 2017;12(3):e0173418

3. Liao S, Dong W, Lv L, Guo H, Yang J, Zhao H, Huang R, Yuan Z, Chen Y, Feng $\mathrm{S}$, et al. Heart regeneration in adult Xenopus tropicalis after apical resection. Cell Biosci. 2017;7:70.

4. Sadek HA, Martin JF, Takeuchi JK, Leor J, Nie Y, Giacca M, Lee RT. Multiinvestigator letter on reproducibility of neonatal heart regeneration following apical resection. Stem Cell Rep. 2014;3(1):1.

5. Porrello ER, Mahmoud Al, Simpson E, Hill JA, Richardson JA, Olson EN, Sadek HA. Transient regenerative potential of the neonatal mouse heart Science. 2011;331(6020):1078-80.

6. Andersen DC, Ganesalingam S, Jensen CH, Sheikh SP. Do neonatal mouse hearts regenerate following heart apex resection? Stem Cell Rep. 2014;2(4):406-13.

7. Bryant DM, O'Meara CC, Ho NN, Gannon J, Cai L, Lee RT. A systematic analysis of neonatal mouse heart regeneration after apical resection. J Mol Cell Cardiol. 2015:79:315-8.

8. Zebrowski DC, Jensen CH, Becker R, Ferrazzi F, Baun C, Hvidsten S, Sheikh SP, Polizzotti BD, Andersen DC, Engel FB. Cardiac injury of the newborn mammalian heart accelerates cardiomyocyte terminal differentiation. Sci Rep. 2017;7(1):8362
9. Hesse M, Welz A, Fleischmann BK. Heart regeneration and the cardiomyocyte cell cycle. Pflugers Archiv. 2018;470(2):241-8.

10. Matrone G, Tucker CS, Denvir MA. Cardiomyocyte proliferation in zebrafish and mammals: lessons for human disease. Cell Mol Life Sci. 2017;74(8):1367-78

11. Jopling C, Sleep E, Raya M, Marti M, Raya A, Izpisua Belmonte JC. Zebrafish heart regeneration occurs by cardiomyocyte dedifferentiation and proliferation. Nature. 2010;464(7288):606-9.

12. Poss KD, Wilson LG, Keating MT. Heart regeneration in zebrafish. Science. 2002;298(5601):2188-90.

13. Chablais $F$, Jazwinska A. The regenerative capacity of the zebrafish heart is dependent on TGF $\beta$ signaling. Development. 2012;139(11):1921-30.

14. Smith SJ, Ataliotis P, Kotecha S, Towers N, Sparrow DB, Mohun TJ. The MLC1 $v$ gene provides a transgenic marker of myocardium formation within developing chambers of the Xenopus heart. Dev Dyn. 2005;232(4):1003-12.

15. Rankin SA, Zorn AM, Buchholz DR. New doxycycline-inducible transgenic lines in Xenopus. Dev Dyn. 2011;240(6):1467-74.

16. Waldner C, Sakamaki K, Ueno N, Turan G, Ryffel GU. Transgenic Xenopus laevis strain expressing cre recombinase in muscle cells. Dev Dyn. 2006;235(8):2220-8.

17. Ito K, Morioka M, Kimura S, Tasaki M, Inohaya K, Kudo A. Differential reparative phenotypes between zebrafish and medaka after cardiac injury. Dev Dyn. 2014;243(9):1106-15.

18. Lai S-L, Marín-Juez R, Moura PL, Kuenne C, Lai JKH, Tsedeke AT, Guenther S, Looso M, Stainier DYR. Reciprocal analyses in zebrafish and medaka reveal that harnessing the immune response promotes cardiac regeneration. eLife. 2017;6:e25605. https://doi.org/10.7554/eLife.25605.

19. Godwin JW, Debuque R, Salimova E, Rosenthal NA. Heart regeneration in the salamander relies on macrophage-mediated control of fibroblast activation and the extracellular landscape. NPJ Regen Med. 2017;2(1):22.

20. Franchini $A$, Bertolotti E. The thymus and tail regenerative capacity in Xenopus laevis tadpoles. Acta Histochem. 2012;114(4):334-41.

21. Franchini A, Bertolotti E. The thymus and skin wound healing in Xenopus laevis adults. Acta Histochem. 2014;116(7):1141-7.
Ready to submit your research? Choose BMC and benefit from:

- fast, convenient online submission

- thorough peer review by experienced researchers in your field

- rapid publication on acceptance

- support for research data, including large and complex data types

- gold Open Access which fosters wider collaboration and increased citations

- maximum visibility for your research: over $100 \mathrm{M}$ website views per year

At $B M C$, research is always in progress.

Learn more biomedcentral.com/submissions 